\title{
Prävention ist die halbe Miete
}

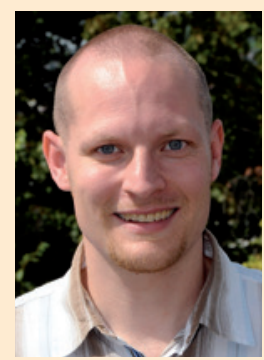

Christian Schäfer, Stuttgart
Das Jahr neigt sich dem Ende zu und die (leider oft stressige) Vorweihnachtszeit ist in Kürze schon wieder vorbei. Es ist meist bald wieder mehr Zeit für etwas Entschleunigung und dafür, sich über die Feiertage mit den Verwandten und Freunden zu treffen. Geht es Ihnen so, dass die guten Vorsätze, die man sich für das vergangene Jahr vorgenommen hat, gerade in dieser besinnlichen Zeit langsam wieder aus den Untiefen des Gedächtnisses ins Bewusstsein gelangen? Und dann kommt die Erkenntnis (die eigentlich schon viel länger da war), dass sich bestenfalls nur ein Bruchteil davon wirklich umsetzen ließ - aus welchen Gründen auch immer. Irgendwie haben sich dann doch wieder zu viele Süßigkeiten in den Einkaufswagen geschlichen und das Wetter war oft nicht passend zum Joggen oder der Schweinehund war wieder einmal zu übermächtig.

Wenn Sie sich hierin wieder erkennen, dann sind Sie in Deutschland sicherlich nicht alleine, wahrscheinlich gehören Sie sogar zur deutlichen Mehrheit. Der vollgepackte und des Öfteren stressige Alltag bringt uns dazu, viele sinnvolle, aber uns eben zusätzlich auferlegte Pflichten und Aufgaben nach ein paar Wochen wieder zu verdrängen. Eine Lösung kann es sein, sich nicht zu viel auf einmal vorzunehmen, sich Etappenziele zu setzen sowie sich für das Erreichen derselben zu belohnen, hierbei nicht zu streng mit sich selbst zu sein und sich die Vorteile eines dauerhaften Wandels immer wieder vor Augen zu führen (z.B. geht man mit einer Reduktion des „Rettungsrings“ auf den Hüften gegen kardiovaskuläre Krankheiten vor und passt wieder besser in die Hosen, die man sich vor einem Jahr gekauft hat).

Ja, so ist das mit den Vorsätzen und der Prävention eben: eine tolle Sache - aber eben nur dann, wenn man die Theorie auch mit Praxis füllen kann. Ist das Nichtgelingen für manche einfach etwas störend, kann das Ausbleiben von präventiven Maßnahmen bei ernsthaft kranken Personen oder solchen, die es zu werden drohen, dramatische Konsequenzen haben. Prävention kann in diesem Fall kurzfristig schon zu entscheidenden Verbesserungen in der Prognose führen. Zum Jahresabschluss habe ich daher zusammen mit den 2 Gasteditoren das Schwerpunktthema „Qualitätssicherung in der Dialyse“ sowie im Supplement das Thema „Ernährung bei Dialysepatienten" geplant. Bei diesen Themengebieten dreht sich vieles darum, die Prognose von Dialysepatienten mithilfe von präventiven Maßnahmen und Behandlungen (eine individuell angepasste Ernährung und Dialysetherapie) zu verbessern.

Natürlich finden Sie in den beiden vorliegenden Heften auch noch viele andere interessante Beiträge. Ich wünsche Ihnen eine besinnliche Lektüre der Dialyse aktuell 10/2014 und des Supplements „Im Fokus“ S1/2014 sowie frohe Weihnachten und einen guten Rutsch ins neue Jahr!

P.S.: Und nehmen Sie sich schon einige, aber nicht zu viele gute Vorsätze für 2015 vor! 